\title{
BMJ Open Multicentre randomised controlled trial to evaluate the efficacy of pre-emptive inferior mesenteric artery embolisation during endovascular aortic aneurysm repair on aneurysm sac change: protocol of Clarify IMA study
}

Shigeo Ichihashi (D) , ${ }^{1}$ Mitsuyoshi Takahara, ${ }^{2}$ Naoki Fujimura, ${ }^{3}$ Satoru Nagatomi, ${ }^{1}$ Shinichi Iwakoshi, ${ }^{1}$ Francesco Bolstad, ${ }^{4}$ Kimihiko Kichikawa ${ }^{1}$

To cite: Ichihashi S, Takahara M, Fujimura N, et al. Multicentre randomised controlled trial to evaluate the efficacy of pre-emptive inferior mesenteric artery embolisation during endovascular aortic aneurysm repair on aneurysm sac change: protocol of Clarify IMA study. BMJ Open 2020;10:e031758. doi:10.1136/ bmjopen-2019-031758

- Prepublication history for this paper is available online. To view these files, please visit the journal online (http://dx.doi org/10.1136/bmjopen-2019031758).

Received 18 May 2019 Revised 26 November 2019 Accepted 03 February 2020
Check for updates

(C) Author(s) (or their employer(s)) 2020. Re-use permitted under CC BY-NC. No commercial re-use. See rights and permissions. Published by BMJ.

For numbered affiliations see end of article.

Correspondence to Dr Shigeo Ichihashi; shigeoichivasc@gmail.com

\section{ABSTRACT}

Introduction Type II endoleak (EL) is frequently seen after endovascular aneurysm repair (EVAR) for abdominal aortic aneurysm (AAA) and is often considered responsible for aneurysm sac enlargement if it persists. In order to reduce type II EL and consequent sac enlargement, pre-emptive embolisation of the inferior mesenteric artery (IMA), which is a main source for persistent type II EL, has been introduced in many vascular centres. At present, there is a lack of robust evidence to support the efficacy of preemptive embolisation of IMA on reduction of persistent type II EL with subsequent sac shrinkage.

Method and analysis This multicentre, randomised controlled trial will recruit 200 patients who have fusiform $\mathrm{AAA} \geq 50 \mathrm{~mm} /$ rapidly enlarging fusiform $\mathrm{AAA}$, with patent IMA, and randomly allocate them either to a pre-emptive IMA embolisation group or non-embolisation control group in a ratio of 1:1. The primary endpoint is the difference of aneurysm sac volume change assessed by CT scans between the pre-emptive IMA embolisation group and the control group at 12 months after EVAR. The secondary endpoints are defined as change of aneurysm sac volume in both groups at 6 and 24 months, freedom from sac enlargement at 12 and 24 months after EVAR, prevalence of type II EL at 1, 6, 12 and 24 months evaluated by contrastenhanced CT, reintervention rate, aneurysm related mortality, overall survival, perioperative morbidity, volume of contrast media used during EVAR and dosage of radiation.

Ethics and dissemination The protocol has been reviewed and approved by the ethics committee of Nara Medical University (No. 2113). The findings of this study will be communicated to healthcare professionals, participants and the public through peer-reviewed publications, scientific conferences and the University Hospital Medical Information Network Clinical Trials Registry home page.

Trial registration number UMIN000035502.

\section{INTRODUCTION}

Endovascular aortic aneurysm repair (EVAR) has become the first-line therapy for abdominal aortic aneurysm (AAA) in developed
Strengths and limitations of this study

- This is a multicentre, randomised controlled trial comparing the effect of pre-emptive embolisation of inferior mesenteric arteries during endovascular aneurysm repair.

- All imaging analysis including volumetry using three-dimensional workstation will be analysed and adjudicated by a core laboratory

- The limitations of this study are the unpredictability of technical success rate in inferior mesenteric artery embolisation.

countries due to its low invasiveness. A major problem after EVAR, however, is a late aneurysm rupture. Especially, after 8 years of EVAR, aneurysm-related death due to sac expansion and following rupture were seen significantly more often than in patients treated through open surgical operations. ${ }^{1}$ Endoleak (EL) is considered a main cause of aneurysm sac expansion. Among ELs, type II EL is seen most frequently, prevalence being $8 \%-45 \%{ }^{2-7}$ Particularly, type II EL from the inferior mesenteric artery (IMA) with a diameter of $2.5 \mathrm{~mm}$ or more, and multiple sources from the IMA and lumber arteries have been reported as contributing factors of persistent type II EL and subsequent sac enlargement. ${ }^{78}$ For cases in which AAA expands due to type II EL, percutaneous embolisation either via a transarterial or translumbar approach are widely performed. However, despite these percutaneous reinterventions, the treatment efficacy is not satisfactory. Long and tortuous collateral vessels can preclude the advancement of microcatheters to aneurysm sacs. An incomplete embolisation of the EL nidus 
could lead to recurrence of type II EL and further sac enlargement, frequently necessitating repetitive reintervention. ${ }^{9-11}$ Following the suboptimal results, many facilities have introduced pre-emptive embolisation of IMA during EVAR. Previous retrospective studies have reported that the pre-emptive embolisation of IMA reduced both the prevalence of type II ELs and the need for second interventions after EVAR by $70 \%$ and $90 \%$, respectively, with a subsequent reduction of the sac expansion rate. ${ }^{12}$ Recently, one randomised controlled trial evaluating the safety and efficacy of the IMA embolisation was published, demonstrating the reduction of type II ELs and the accelerated sac shrinkages in the embolisation arm. ${ }^{13}$ However, the study was conducted in a single centre, and sac size change was evaluated by diameter measurement without core lab adjudication, raising concern of biased evaluation. Under the circumstances, the Clarify IMA, multicentre, core lab adjudicated, randomised controlled study has thus been planned to demonstrate the clinical safety and efficacy of pre-emptive embolisation of IMA versus non-embolisation of IMA during EVAR for AAA. Three-dimensional (3D) volumetric analysis will be introduced to evaluate the aneurysm sac change objectively.

\section{MATERIALS AND METHODS}

The Standard Protocol Items: Recommendations for Interventional Trials checklist has been used to report the trial's design, conduct, analysis and interpretation and to assess the validity of its results. ${ }^{14}$ The trial has been registered in UMIN Clinical Trials Registry (CTR) (table 1).

\section{Design of the study}

The Clarify IMA trial is a prospective, parallel group, open-label, multicentre, randomised controlled study in Japan. After baseline assessment, all participants will be randomly allocated to either the IMA embolisation group or the control group in a 1:1 ratio. The outcomes among both groups will be followed at 1, 6, 12 and 24 months after EVAR. All randomised patients will be included in the analysis based on the intention-to-treat principle.

\section{Study objectives and endpoints}

Primary objectives

The primary objective of the Clarify IMA study is to compare the sac size after EVAR between pre-emptive embolisation of IMA during EVAR and non-embolisation of IMA.

\section{Primary endpoint}

The study is powered based on the difference of aneurysm sac volume change assessed by CT between the preemptive IMA embolisation group and the control group at 12 months after EVAR.

\section{Secondary objectives}

The secondary objectives are to assess the safety, efficacy and economic impact of the pre-emptive embolisation of IMA.

\section{Secondary endpoint}

The following secondary endpoints will be evaluated.

Aneurysm sac volume change in both groups at 6 , and 24 months.

Aneurysm sac diameter change in both groups at 6, 12 and 24 months.

Freedom from sac enlargement at 6, 12 and 24 months after EVAR*

Prevalence of type II EL at 1, 6, 12 and 24 months evaluated by contrast-enhanced CT (CECT).

Reintervention rate.

Aneurysm related mortality.

Overall survival.

Perioperative complication.

Volume of contrast media used during EVAR.

Dosage of X-ray.

Operation time.

Medical expenses.

*Enlargement of the aneurysm sac volume is defined as a volume increase of at least $2 \%$ or $5 \mathrm{~mm}$ in minor axis on the transaxial plane.

\section{Participants recruitment}

All procedures will be conducted at 20-30 institutes including academic and community hospitals in Japan. Vascular specialists including vascular surgeons, interventional radiologists and cardiologists at each site will determine the eligibility of patients for the study according to the following inclusion and exclusion criteria.

\section{Inclusion criteria}

The patient is/has:

1. Age $\geq 20$ at the time of informed consent signature.

2. An informed consent form signed by subject.

3. Diameter of IMA $\geq 2.5 \mathrm{~mm}$ (measurement at the largest point).

4. Fusiform AAA with diameter $\geq 50 \mathrm{~mm}$ or rapid enlargement of sac $\geq 5 \mathrm{~mm}$ in diameter within 6 months.

5. Proximal neck diameter of $\leq 28 \mathrm{~mm}$.

6. Proximal neck length $\geq 15 \mathrm{~mm}$.

7. Infrarenal angulation $\leq 60^{\circ}$ (reasonable effort).

\section{Exclusion criteria}

The patient is/has:

1. Hereditary disease such as Marfan syndrome, EhlersDanlos syndrome.

2. Pregnant female, female with possible pregnancy, within postpartum 28 days, during breast feeding.

3. Life expectancy $<1$ year or advanced stage of malignant tumour.

4. Isolated iliac aneurysm.

5. Mycotic aneurysm.

6. Saccular aneurysm.

7. Penetrating atherosclerotic ulcer .

8. Renal insufficiency with estimated glomerular filtration rate $\leq 30 \mathrm{~mL} / \mathrm{min} / 1.73 \mathrm{~m}^{2}$ and has not introduced dialysis.

9. Significant stenosis or occlusion at the ostium of IMA. 
Table 1 Trial registration data

\section{Data category}

Primary registry and trial identifying no

Date of registration in primary registry

Source(s) of monetary or material support

Primary sponsor

Contact for public queries

Contact for scientific queries

Public title

Scientific title

Countries of recruitment

Health condition(s) or problem(s) studied

Intervention(s)

Inclusion criteria

\section{Information}

UMIN Clinical Trials Registry (CTR): UMIN000035502

1 February 2019

W.L.Gore \& Associates, Terumo, Boston Scientific Japan

W.L.Gore \& Associates, Terumo, Boston Scientific Japan

SI, MD, email: ichihash@naramed-u.ac.jp

$\mathrm{SI}, \mathrm{MD}$

\section{Efficacy of pre-emptive IMA embolisation during EVAR}

A multicentre randomised controlled trial to evaluate the efficacy of pre-emptive IMA embolisation during EVAR on aneurysm sac change: Clarify IMA

Japan

Aneurysm sac change, type II endoleak, IMA

Active comparator: IMA embolisation

Comparator: No embolisation

Age $\geq 20$ at the time of informed consent signature.

An Informed consent form signed by subject.

Diameter of IMA $\geq 2.5 \mathrm{~mm}$ (measurement at the largest point)

Fusiform AAA with diameter $\geq 50 \mathrm{~mm}$ or rapid enlargement of sac $\geq 5 \mathrm{~mm}$ in diameter within 6 months

Proximal neck diameter of $\leq 28 \mathrm{~mm}$

Proximal neck length $\geq 15 \mathrm{~mm}$

Infrarenal angulation $\leq 60^{\circ}$ (reasonable effort)

Exclusion criteria

Hereditary disease such as Marfan syndrome, Ehlers-Danlos syndrome

Pregnant female, female with possible pregnancy, within postpartum 28 days, during breast feeding

Life expectancy $<1$ year or advanced stage of malignant tumour

Isolated iliac aneurysm

Mycotic aneurysm

Saccular aneurysm

Penetrating atherosclerotic ulcer

Renal insufficiency with eGFR $\leq 30 \mathrm{~mL} / \mathrm{min} / 1.73 \mathrm{~m}^{2}$ and has not introduced dialysis

Significant stenosis or occlusion at the ostium of IMA

IMA originates from proximal neck of the abdominal aneurysm

Significant stenosis or occlusion in the celiac and superior mesenteric arteries

History of anaphylactic shock due to iodine contrast media

Study type

Interventional

Allocation: randomised; Intervention model: parallel assignment; Masking: open label

Primary purpose: prevention

\begin{tabular}{ll} 
Date of first enrolment & Unenrolled \\
\hline Target sample size & 200 \\
Recruitment status & Preinitiation \\
Primary outcome(s) & Difference of aneurysm sac volume change assessed by CT between pre-emptive IMA \\
& embolisation group and control group at 12 months after EVAR \\
Key secondary outcomes & Aneurysm sac volume change in both groups at 6 , and 24 months \\
& Aneurysm sac diameter change in both group at 6,12 and 24 months \\
& Freedom from sac enlargement at 6,12 and 24 months after EVAR \\
& Prevalence of type II EL at $1,6,12$ and 24 months evaluated by CECT \\
& Reintervention rate \\
& Aneurysm-related mortality \\
& Overall survival \\
& Perioperative complication \\
& Volume of contrast media used during EVAR \\
Dosage of $X-$ ray & Operation time \\
& Medical expenses
\end{tabular}

AAA, abdominal aortic aneurysm; CECT, contrast-enhanced CT; CTR, Clinical Trials Registry; eGFR, estimated glomerular filtration rate; EVAR, endovascular aneurysm repair; IMA, inferior mesenteric artery; UMIN, University Hospital Medical Information Network. 
10. IMA originates from the proximal neck of the abdominal aneurysm.

11. Significant stenosis or occlusion in the celiac and superior mesenteric arteries.

12. History of anaphylactic shock due to iodine contrast media.

\section{Randomisation, masking}

Participants will be randomised using CapTool Lite (Mebix Tokyo, Japan), which is an independent, online, central randomisation service. The randomisation will be performed using a dynamic allocation method based on the centre and AAA diameter. Allocation concealment will be guaranteed by use of a central randomisation system via the internet. In the system, the codes will be kept by an independent allocator. A random component generated by computer will be used. The service will not release the result of allocation until the patient is recruited and registered into the trial, which takes place after all baseline measurements have been completed. The individual results of allocation will be open to his/her care providers and the participant, but not to outcome assessors.

\section{Data forms and data entry}

In the current study, all data will be entered via internet website. This will be done at the participating hospitals. Data integrity will be enforced through a variety of mechanisms. Referential data rules, valid values, range checks and consistency checks against data already stored in the database will be supported.

\section{Interventions}

For the patients allocated to IMA embolisation group, embolisation of IMA will be performed using either metallic coils or vascular plugs. In order to improve the technical success rate of IMA embolisation, participating physicians will be selected from those who are experienced in the procedures. Regardless of the group the patient is allocated to, embolisation of lumber, sacral arteries is prohibited. Injection of liquid embolisation material such as n-butyl-2-cyanoacrylate/Onix into aneurysm sac during EVAR is also prohibited. Commercially available stentgrafts in Japan will be used for the EVAR.

\section{Medications}

Antiplatelet and/or anticoagulation therapy will be employed at the discretion of physicians depending on the patients' comorbidities. Administration of tranexamic acid, an antifibrinolytic agent, for the purpose of accelerating thrombosis of the aneurysm sac, is forbidden during the study period as the therapy can influence the prevalence of EL or aneurysm sac change. ${ }^{15}$

\section{Participant timeline}

All patients randomised into the clinical investigation will have clinical follow-ups and CECT scans within 4 weeks postprocedure, 6, 12 and 24 months postprocedure \pm 2 months (table 2). Adherence to the protocol will be reinforced by on-site training and newsletters.

\section{CT examination}

Triple phase CT scan (plain, arterial phase, venous phase) will be conducted to evaluate the presence of type II EL and sac volume. CT performed within 1 month after EVAR is defined as the baseline CT.

\section{Image analysis including volumetry of aneurysm sac and EL}

All CT image data will be collected at the department of Radiology, Nara Medical University. Three technologists who have more than 10 years of experience conducting CT angiography and have expertise using 3D workstation will be evaluating the presence of EL, type of EL and measuring aneurysm sac volume. The aortic volume will be measured in a semiautomated manner using SYNAPSE VINCENT (FUJIFILM Corporation, Tokyo, JAPAN).

\section{Reintervention}

Endovascular or surgical intervention will be considered if the aneurysm sac expands $\geq 5 \mathrm{~mm}$ in minor axis on any follow-up CT compared with the baseline CT or if stentgraft migration or dislodgement occurs with/without

Table 2 Schedule of participants visit and data collection

\begin{tabular}{|c|c|c|c|c|}
\hline & Baseline & Procedure & Within 4 weeks & $6,12,24$ months ( \pm 2 month) \\
\hline Patient medical/clinical history & * & & & \\
\hline CT scan & * & & * & * \\
\hline Blood exam (eGFR) & * & & * & * \\
\hline DSA & & * & & \\
\hline Second intervention & & & * & * \\
\hline $\begin{array}{l}\text { Adverse events/device deficiencies/adverse } \\
\text { product experiences }\end{array}$ & & * & * & * \\
\hline
\end{tabular}

DSA, digital subtraction angiography; eGFR, estimated glomerular filtration rate. 
type I or type III EL. Any reintervention procedures will be performed at the discretion of physicians.

\section{Participant retention}

Once a patient is randomised, the study site will make every reasonable effort to follow the patient for the entire study period. It is projected that the rate of lost to-follow-up on an annual basis will be at most $15 \%$.

\section{Sample size calculation}

The current clinical trial will enrol a total of 200 patients. Our null hypothesis is that CT-evaluated aneurysm sac volume change will not be different (ie, will be equal) between the pre-emptive IMA embolisation group and the control group at 12 months after EVAR. A previous report ${ }^{16}$ reported that the mean change \pm SD was $30.3 \% \pm 21.8 \%$ in patients with an occluded IMA versus $16.2 \% \pm 19.2 \%$ in patients with a patent IMA. To be conservative, we hypothesise that the mean CT-assessed aneurysm sac volume change at 12 months will be $30 \%$ after EVAR with IMA embolisation versus $16 \%$ after EVAR without IMA embolisation, with the SD equal to $22 \%$. We also hypothesise that IMA will be successfully embolised in $80 \%$ of patients allocated to the IMA embolisation group; the rest $20 \%$ of the population will have the outcome measure equivalent to the control group. We also hypothesise that $15 \%$ of the study participants will drop out within 1 year. Furthermore, the dynamic allocation will not guarantee the equivalence in number of participants between the two groups; a 5000-time simulation showed that between-group difference in number of participants will be distributed with the median equal to six. Taking all the above information into consideration, we conducted a 10 000-time simulation for statistical power, using $\mathrm{R}$ software. Consequently, we confirmed that 200 people in total will be sufficient to detect the intergroup difference of the sac volume change with a two-sided significance level (alpha) of $5 \%$ and a power ( 1 -beta) of more than $90 \%$. The anticipated accrual rate is approximately 10 subjects per month for a total accrual period of approximately 20-30 months.

Sample size sensitivity: This sample size will also have $>80 \%$ power to detect the intergroup difference of the sac volume change, either when the mean aneurysm sac volume change is $28 \%$ in the IMA embolisation group, when the mean aneurysm sac volume change is $18 \%$ in the control group, when the SD of the aneurysm sac volume change is $25 \%$, or when IMA will be successfully embolised in $70 \%$ of patients allocated to the IMA embolisation group.

\section{Statistical analysis}

According to the intention-to-treat principle, all randomised patients will be included in this analysis. The primary endpoint, that is, the CT-assessed aneurysm sac volume at 12 months, is expected to follow a normal distribution, or at least a symmetric bell-shaped one, ${ }^{16}$ and the intergroup difference will be tested using the
Welch's t-test. We adopt the Welch's t-test rather than the Student's t-test, simply because we do not assume the equality of the variance (or SD) between the two groups, and we want to be robust even if the variance is different between the groups. Freedom from reintervention, freedom from aneurysm-related death, and overall survival, all of which can be regarded as right-censored data, will be evaluated using the Kaplan-Meier estimate. Their intergroup difference will be tested using the logrank test. On the other hand, the sac enlargement (or freedom from it) and type II EL cannot be confirmed until image examination is performed; they would be overlooked if the examination is not done. In this sense, these data are not right censored but interval censored, and are not suitable for the Kaplan-Meier estimation, which is designed for right-censored data. We will, therefore, assess these outcomes as the proportion at each follow-up visit, and will test their intergroup difference using the $\chi^{2}$ test. The proportion of perioperative complications will also be compared by the $\chi^{2}$ test. X-ray radiation dose (Gy), volume of contrast media used and operation time for endovascular procedures will be compared between the groups using a non-parametric statistical test. We will perform a complete-case analysis (without imputing missing data) as the primary analysis, and will apply the multiple imputation method as a sensitivity analysis. Furthermore, we will perform a secondary analysis with adjustment for baseline characteristics including concomitant medications, if we find significant differences between the two groups.

\section{Potential influence of reintervention on the primary outcome measure}

Reintervention will have a potential influence on the size of the aneurysm sac. A previous study ${ }^{17}$ reported that only $9 \%$ of the sacs shrank after reinterventions. Another report also demonstrated that sac expansion was prevented only in $44 \%$ of the cases. ${ }^{11}$ Indeed, sac enlargement after reintervention is commonly seen in clinical practice. We will, therefore, determine the primary outcome measure (the size of the aneurysms sac) as the larger of the measurement right before reintervention and that at 12 months in cases undergoing reintervention. Of course, we recognise that this determination will still be subject to the underestimation in reintervention cases (the sac would become larger if reintervention were not performed). We, therefore, plan to perform the following sensitivity analyses: adopting the Bayesian multiple imputation, and developing a parametric accelerated failure time model (parametric survival regression model) based on a Gaussian distribution. Both sensitivity analyses will be under the hypothesis the true value would be larger than the measured one (ie, censored at the measured value). On the other hand, reintervention for sac enlargement within 1 year is rare in clinical practice, ${ }^{18}$ and we believe that the influence on the overall outcome would be small in practical terms, if any. 


\section{Data monitoring}

Healthcare providers, independent of investigators, will review the study plan compliance, adherence to the protocol and data quality. Electronic data capture system and medical record/imaging data will be compared with ensure that the study is being conducted in compliance with pertinent regulatory requirements. On-site data monitoring will be performed for $20 \%$ of the cases enrolled. Adverse events, all unwanted or unintended diseases or disorders that occur in the study subject during the study period, as well as their symptoms will be communicated to the principal investigators from investigational sites.

\section{Ethics and dissemination}

An informed consent will be obtained from every subject. Physicians will give information sheets and consent forms, and will discuss the trial with patients regarding the objectives of the study, and potential advantages and disadvantages of the IMA embolisation. All modifications to the protocol will be reported to the University Hospital Medical Information Network Clinical Trials Registry (UMIN-CTR) and communicated to the public (UMIN000035502). To assure confidentiality, all CT data, which will be collected at Nara Medical university, will be coded by identification number without personal information and stored at Nara Medical University School of Medicine in locked cabinets. Electronic data will be stored on a secure password-protected server during the study. Private insurance for clinical trials will provide cover for non-negligent harm associated with the protocol. The findings of this study will be communicated to healthcare professionals, participants and the public through peerreviewed publications, scientific conferences and the UMIN-CTR home page.

\section{Patient and public involvement statement}

patients and the public will not be involved in the design, conduct and reporting of the research.

\section{DISCUSSION}

Type II EL, if it persists longer than 6 months, has been reported to be responsible for sac enlargement after EVAR. ${ }^{19} 20$ Accordingly, multiple vascular centres have investigated anatomical risk factors of persistent type II EL and introduced a pre-emptive embolisation of IMA protocol during EVAR for high-risk patients. The anatomical risk factors for developing persistent type II EL include patent IMA, higher number of patent lumber arteries and the diameter of IMA and lumber artery. ${ }^{821-23}$ Regarding the diameter of IMA, patients whose diameter of IMA $\geq 2.5 \mathrm{~mm}$ will be recruited to this study as Fukuda et al demonstrated the highest diagnostic performance to predict persistent type II EL occurred when a $2.5 \mathrm{~mm}$ threshold was chosen. ${ }^{8}$ On the other hand, inclusion/ exclusion criteria will not be defined for lumber arteries because of the following reasons, (1) evaluation of patency of lumber arteries only by CTA would be challenging because the diameter of lumber arteries is usually smaller than IMA, (2) consideration of both number of patent lumber arteries and diameter would make the study protocol complicated and is not widely applicable in every participating hospital. In order to evaluate the simple effect of IMA embolisation, embolisation of lumber arteries will be prohibited in the study.

Violation of anatomical regulations in the device instructions provided by each stent graft manufacture, particularly in proximal neck, has been proven to be associated with a higher risk of sac enlargement after EVAR. ${ }^{24-26}$ To minimise the risk of type II EL or endotension and evaluate an unmixed influence of IMA embolisation on the aneurysm sac, the following generalised anatomical criteria for the proximal neck will be applied in this study; neck length $\geq 15 \mathrm{~mm}$, infrarenal neck angulation $\leq 60^{\circ}$, neck diameter $\leq 28 \mathrm{~mm}$.

Sac size change will be evaluated by volumetry analysis. Even though sac diameter is easy to measure and commonly used, its reproducibility is not high and it is likely that it occurs when the upper part of the aneurysm enlarges while the lower part shrinks or vice versa. Aneurysm volume measurements will be performed by experienced technologists independent of investigators, enabling the influence of IMA embolisation of aneurysm sacs to be measured objectively. Enlargement of the aneurysm sac volume will be defined as a volume increase of at least $2 \%$, as previously reported surveillance protocols after EVAR have identified a $2 \%$ vol increase as a significant endpoint and the degree of volume variability is less than $2 \%$ for experienced, well trained 3D technologists. $^{2728}$

There are several limitations in the study. First, the technologists at core lab who will perform image analysis cannot be completely blinded because metallic artefacts can arise from coils or plugs used for IMA embolisation. Volumetric analysis, instead of diameter analysis should minimise the observer bias and data monitoring will be implemented on $20 \%$ of all the measurements at core lab. Second, the technical success of IMA embolisation, which is define as disappearance of antegrade flow of IMA after embolisation, cannot be predicted before the initiation of the study, however, all vascular specialists participating in the study have high levels of expertise in endovascular procedures and each participating physician is strongly recommended to perform at least three cases of IMA embolisation prior to initiation of the enrolment. Finally, when IMA originates above the aneurysm sac, deployment of stent graft can obstruct the IMA orifice even without embolisation of IMA, and can underestimate the effect of IMA embolisation.

\section{Author affiliations}

${ }^{1}$ Radiology, Nara Medical University, Kashihara, Japan

${ }^{2}$ Department of Diabetes Care Medicine and Department of Metabolic Medicine, Osaka University, Suita, Osaka, Japan

${ }^{3}$ Vascular Surgery, Saiseikai Central Hospital, Minato-ku, Tokyo, Japan

${ }^{4}$ Clinical English, Nara Medical University, Kashihara, Japan 
Contributors SI conceived of the study. SI, NF and SN initiated the design of the study and MT, SI, FB and KK helped with implementation. SI, MT, NF and FB prepared the first draft of the manuscript. MT provided statistical expertise in clinical trial design and is conducting the primary statistical analysis. All authors contributed to the refinement of the study protocol and approved the final manuscript.

Funding The study will have financial support from manufacturers of stentgrafts or embolic agents: W. L. Gore and Associates (Flagstaff, Arizona, USA), Terumo (Tokyo, Japan), and Boston Scientific Japan (Tokyo, Japan). The design, management, analysis and reporting of the study are entirely independent of these manufactures.

Competing interests NF serves as a consultant to W.L. Gore, Cook Medical and Endologix.

Patient consent for publication Not required.

Ethics approval The protocol has been reviewed and approved by the ethics committee of Nara Medical University (No. 2113). The protocol issue date is 7 December 2018 and protocol version number is 01 .

Provenance and peer review Not commissioned; externally peer reviewed.

Open access This is an open access article distributed in accordance with the Creative Commons Attribution Non Commercial (CC BY-NC 4.0) license, which permits others to distribute, remix, adapt, build upon this work non-commercially, and license their derivative works on different terms, provided the original work is properly cited, appropriate credit is given, any changes made indicated, and the use is non-commercial. See: http://creativecommons.org/licenses/by-nc/4.0/.

\section{ORCID iD}

Shigeo Ichihashi http://orcid.org/0000-0003-1809-4079

\section{REFERENCES}

1 Patel R, Sweeting MJ, Powell JT, et al. Endovascular versus open repair of abdominal aortic aneurysm in 15-years' follow-up of the UK endovascular aneurysm repair trial 1 (EVAR trial 1): a randomised controlled trial. Lancet 2016;388:2366-74.

2 White GH, Yu W, May J. Endoleak--a proposed new terminology to describe incomplete aneurysm exclusion by an endoluminal graft. $J$ Endovasc Surg 1996;3:124-5.

3 van Marrewijk C, Buth J, Harris PL, et al. Significance of endoleaks after endovascular repair of abdominal aortic aneurysms: the EUROSTAR experience. J Vasc Surg 2002;35:461-73.

4 Baum RA, Stavropoulos SW, Fairman RM, et al. Endoleaks after endovascular repair of abdominal aortic aneurysms. J Vasc Interv Radiol 2003;14:1111-7.

5 Alerci M, Giamboni A, Wyttenbach R, et al. Endovascular abdominal aneurysm repair and impact of systematic preoperative embolization of collateral arteries: endoleak analysis and long-term follow-up. J Endovasc Ther 2013;20:663-71.

6 Ward TJ, Cohen S, Fischman AM, et al. Preoperative inferior mesenteric artery embolization before endovascular aneurysm repair: decreased incidence of type II endoleak and aneurysm sac enlargement with 24-month follow-up. J Vasc Interv Radiol 2013;24:49-55

7 Müller-Wille R, Schötz S, Zeman F, et al. CT features of early type II endoleaks after endovascular repair of abdominal aortic aneurysms help predict aneurysm sac enlargement. Radiology 2015;274:906-16.

8 Fukuda T, Matsuda H, Sanda Y, et al. Ct findings of risk factors for persistent type II endoleak from inferior mesenteric artery to determine indicators of preoperative IMA embolization. Ann Vasc Dis 2014;7:274-9.
9 Walker J, Tucker L-Y, Goodney P, et al. Type II endoleak with or without intervention after endovascular aortic aneurysm repair does not change aneurysm-related outcomes despite sac growth. $J$ Vasc Surg 2015;62:551-61.

10 Haq I-U, Kelay A, Davis M, et al. Ten-year single-centre experience with type II endoleaks: intervention versus observation. Vasc Med 2017;22:316-23.

11 Sarac TP, Gibbons C, Vargas L, et al. Long-term follow-up of type II endoleak embolization reveals the need for close surveillance. J Vasc Surg 2012;55:33-40.

12 Manunga JM, Cragg A, Garberich R, et al. Preoperative inferior mesenteric artery embolization: a valid method to reduce the rate of type II endoleak after EVAR? Ann Vasc Surg 2017;39:40-7.

13 Samura M, Morikage N, Otsuka R, et al. Endovascular aneurysm repair with inferior mesenteric artery embolization for preventing type II endoleak: a prospective randomized controlled trial. Ann Surg 2020;271:238-44.

14 Chan A-W, Tetzlaff JM, Gøtzsche PC, et al. Spirit 2013 explanation and elaboration: guidance for protocols of clinical trials. BMJ 2013;346:e7586.

15 Aoki A, Suezawa T, Yamamoto S, et al. Effect of antifibrinolytic therapy with tranexamic acid on abdominal aortic aneurysm shrinkage after endovascular repair. J Vasc Surg 2014;59:1203-8.

16 Kray J, Kirk S, Franko J, et al. Role of type II endoleak in sac regression after endovascular repair of infrarenal abdominal aortic aneurysms. J Vasc Surg 2015;61:869-74.

17 Ogawa $\mathrm{Y}$, Nishimaki $\mathrm{H}$, Osuga $\mathrm{K}$, et al. A multi-institutional survey of interventional radiology for type II endoleaks after endovascular aortic repair: questionnaire results from the Japanese Society of endoluminal metallic stents and grafts in Japan. Jpn J Radiol 2016;34:564-71.

18 Fujimura N, Obara H, Matsubara K, et al. Characteristics and risk factors for type 2 endoleak in an East Asian population from a Japanese multicenter database. Circ J 2016;80:118-23.

19 Higashiura W, Greenberg RK, Katz E, et al. Predictive factors, morphologic effects, and proposed treatment paradigm for type II endoleaks after repair of infrarenal abdominal aortic aneurysms. $J$ Vasc Interv Radiol 2007;18:975-81.

20 Hoshina K, Ishimaru S, Sasabuchi Y, et al. Outcomes of endovascular repair for abdominal aortic aneurysms: a nationwide survey in Japan. Ann Surg 2019;269:564-73.

21 Samura M, Morikage N, Mizoguchi T, et al. Identification of anatomical risk factors for type II endoleak to guide selective inferior mesenteric artery embolization. Ann Vasc Surg 2018;48:166-73.

22 Guo Q, Du X, Zhao J, et al. Prevalence and risk factors of type II endoleaks after endovascular aneurysm repair: a meta-analysis. PLoS One 2017;12:e0170600.

23 Güntner O, Zeman F, Wohlgemuth WA, et al. Inferior mesenteric arterial type II endoleaks after endovascular repair of abdominal aortic aneurysm: are they predictable? Radiology 2014;270:910-9.

24 Schanzer A, Greenberg RK, Hevelone N, et al. Predictors of abdominal aortic aneurysm sac enlargement after endovascular repair. Circulation 2011;123:2848-55.

25 Waasdorp EJ, de Vries J-PPM, Hobo R, et al. Aneurysm diameter and proximal aortic neck diameter influence clinical outcome of endovascular abdominal aortic repair: a 4-year EUROSTAR experience. Ann Vasc Surg 2005;19:755-61.

26 Leurs LJ, Kievit J, Dagnelie PC, et al. Influence of infrarenal neck length on outcome of endovascular abdominal aortic aneurysm repair. J Endovasc Ther 2006;13:640-8.

27 Caldwell DP, Pulfer KA, Jaggi GR, et al. Aortic aneurysm volume calculation: effect of operator experience. Abdom Imaging 2005;30:259-62.

28 Bley TA, Chase PJ, Reeder SB, et al. Endovascular abdominal aortic aneurysm repair: nonenhanced volumetric CT for follow-up. Radiology 2009;253:253-62. 